\title{
Preferencias para el turismo de aventura en la elaboración de un paquete turístico: Caso Santa Elena, Ecuador
}

\section{Preferences for adventure tourism in the preparation of a tourism package: Case of Santa Elena, Ecuador}

Wilmer Carvache-Franco ${ }^{1}$, Mauricio Carvache-Franco ${ }^{2}$, Orly Carvache-Franco ${ }^{3}$, Xavier Recalde-Lino ${ }^{4}$

Resumen: Las preferencias para el turismo de aventura en un destino traen información importante para elaborar paquetes turísticos y mejorar la oferta de productos en un destino que cuenta con recursos naturales para el desarrollo de esta modalidad de turismo. El presente trabajo empírico se basó en un cuestionario que pretende encontrar un modelo para la creación de paquetes de turismo de aventura. Los resultados evidencian que las preferencias acerca del turismo de aventura en la provincia de Santa Elena muestran el potencial de este tipo de turismo. Los visitantes en este destino prefieren, principalmente, realizar el buceo y la práctica de snorkel, seguido de parapente; luego continúan esquí acuático y canopy. Los resultados contribuirán a crear productos acorde a la demanda del turismo de aventura. Las preferencias determinan que los visitantes desearían estar entre tres días y dos noches, y de dos días con una noche con las modalidades que obtuvieron más porcentaje tales como buceo, snorkel, parapente, canopy y esquí acuático, basado en el precio entre 100 a 150 dólares por paquete turístico, lo cual es accesible para este segmento.

Palabras clave: Preferencias, turismo de aventura, paquetes turísticos, demanda del turismo.
Abstract: The preferences for adventure tourism in a destination provide important information for making tourism packages and improving the supply of products at a destination with the natural resources to undertake this type of tourism. This empirical study was based on a questionnaire that endeavors to find a model for the creation of adventure tourism packages. The results reveal that the preferences for adventure tourism in the province of Santa Elena show the potential for this type of tourism. The visitors to this destination mainly prefer to practice scuba diving and snorkeling, followed by paragliding and then water skiing and canopy. The results will contribute to creating products according to the demand for adventure tourism. The preferences determine that the visitors would like to stay for between three days and two nights, and two days and one night with the arrangements that obtained the highest percentages, such as scuba diving, snorkeling, paragliding, canopy and water skiing based on the price between 100 and 150 dollars per package, which is accessible for this segment.

Key words: Preferences, adventure tourism, tourism packages, tourist demand.

\section{(Presentado: 29 de septiembre de 2017. Aceptado: 27 de noviembre de 2017)}

\footnotetext{
${ }^{1}$ Master Internacional de Turismo, Universidad de Las Palmas de Gran Canarias, España. Profesor e Investigador, Facultad de Ingeniería Marítima, Ciencias Biológicas, Oceánicas y Recursos Naturales, Escuela Superior Politécnica del Litoral (ESPOL), Guayaquil, Ecuador. E-mail: wcarvachqespol.edu.ec

${ }^{2}$ Máster en Dirección de Marketing y Distribución Comercial, Universidad Autónoma de Barcelona, España; Máster en Gerencia de Marketing, Universidad Católica Santiago de Guayaquil, Ecuador. E-mail: scarvachefqunemi.edu.ec.

${ }^{3}$ MBA, Quebec University, Canadá. Universidad Católica Santiago de Guayaquil, Facultad de Especialidades Empresariales. E-mail: orly.carvache\&cu.ucsg.edu.ec

${ }^{4}$ Licenciado en Turismo, Escuela Superior Politécnica del Litoral (ESPOL), Facultad de Ingeniería Marítima, Ciencias Biológicas Oceánicas y Recursos Naturales, Ecuador. E-mail: xrecaldęespol.edu.ec
} 


\section{INTRODUCCIÓN}

El turismo de aventura comienza a desarrollarse como consecuencia de un acercamiento por parte de un mercado turístico que demanda la realización de actividades con cierto nivel de riesgo en entornos naturales (Díaz, 2012). Dichas actividades se realizan fuera de espacios urbanos donde existen entornos naturales con equipos para realizar las modalidades y donde requieren de esfuerzo físico y energía para realizarlos. Siguiendo a Díaz (2012), la amplia gama de productos de turismo de aventura es resultado no sólo de la diversidad geográfica, sino también son consecuencia del perfil del turista; pues en el turismo de aventura no solamente tiene cabida el turismo de perfil marcadamente aventurero (turista duro) que afronta desafíos extremos, sino que también se presenta un tipo de turista (suave) que demanda la realización de actividades con un nivel de riesgo muy controlado. Se puede apreciar que el turismo de aventura apunta no solamente a un segmento de mercado tal como el turista duro, sino también a aquel turista que no tiene conocimiento pero que puede realizarlo o siente ganas de querer hacerlo que sería el turista suave. En esta línea, como señalan Cloke y Perkins (1998), el diseño de actividades de aventura para un turista de perfil suave ha sido una decisión tomada por las empresas ante la necesidad de cubrir una mayor cuota de mercado y la creciente demanda por este tipo de actividades. Así Zamorano (2007), define al turismo de aventura donde algunos lo llaman turismo deportivo, turismos de adrenalina o bien turismo de retos.

Visto de esta forma para The Adventure Travel Trade Association, que es una asociación internacional para la industria del turismo de aventura con miembros en 84 países a nivel mundial, en la publicación de la revista Global Report on Adventure Tourism patrocinada por la World Tourism Organization (UNWTO) en el año 2014, define al turismo de aventura como "Un viaje en que incluye al menos dos de los tres siguientes elementos: Actividad física, ambiente natural e inmersión cultural. Este también puede ser doméstico o internacional y como todo viaje incluye una estadía de no más de un año (Tourism Adventure- Global Report, 2014).

En este contexto, en la provincia de Santa Elena durante los últimos años se han desarrollado algunas modalidades de turismo de aventura que se promocionan a través de folletos turísticos, redes sociales o portales Web. Existen lugares donde se pueden realizar actividades de turismo de aventura como Salinas, San Pablo, Dos Mangas, Ayangue, San Pedro, Montañita y Olón. En estos momentos muchos turistas viajan a la provincia de Santa Elena para disfrutar de actividades de aventura en un entorno de soly playa; pero no se conoce sus preferencias para la creación de paquetes turísticos en este tipo de modalidad de turismo. Este estudio se propone determinar las preferencias del turismo de aventura en la provincia de Santa Elena, para lo cual el presente artículo se compone de una introducción, revisión de la literatura, ubicación del área de estudio, metodología, resultados y discusión y culminando con las conclusiones.

\section{REVISIÓN DE LITERATURA}

Los inicios del turismo de aventura se encuentran muy relacionados con la realización de actividades recreativas de aventura como pasatiempo; una relación que pudo haber contribuido a generalizar la idea de una práctica del turismo de aventura por parte de un turista con amplia experiencia en la actividad desarrollada (Buckley, 2009). Sin embargo, como señalan Cloke \& Perkins (1998) el diseño de actividades de aventura para un turista de perfil suave ha sido una decisión tomada por las empresas ante la necesidad de cubrir una mayor cuota de mercado y la creciente demanda por este tipo de actividades. De este modo Williams (2009) señala que últimamente la demanda de turismo de aventura ha ido creciendo significativamente y examinó empíricamente las relaciones entre valor, satisfacción e intenciones de comportamiento en un contexto de turismo de aventura. Por otra parte, Fletcher (2010) indica que el turismo de aventura abarca una complejidad de cosas que unen la construcción de un secreto público donde el turista mantiene, simultáneamente, el dilema entre el riesgo y la seguridad al momento de realizar las modalidades. Los secretos públicos abarcan tres dimensiones. La primera donde los proveedores y clientes contribuyen simultáneamente al tema de riesgo y no riesgo, del todo en el paquete; la segunda abarca entre los proveedores y turistas, quienes hablan si el viaje es peligroso o seguro a la vez; la tercera abarca en que ambos opinan sobre el viaje, algunos lo consideran de manera irrelevante, manteniendo incompatibilidad entre todos. El 
autor concluyó que el turismo de aventura basado en su análisis abarca todo desde la compra hasta la realización incluso como el guía interactúa y se preocupa por la incógnita del turista si es seguro o riesgoso.

De esta manera, Cole (2016) en base a tours de ciclismo de montaña establece que las firmas que ofrecen el ciclismo de aventura están bien definidas en cuanto a variables de costo, estilo de conducción, duración del recorrido, habilidad y aptitud. Así, Saldaña (2014) señala cuales serían los elementos que identificarían la calidad técnica en diferentes regiones donde se desarrolla el turismo de aventura que son: Seguridad, medio ambiente, entidad, cliente, recursos humanos, actividad y recursos materiales; considerando que el parámetro de que más debe tomar en cuenta sería la seguridad ya que se debe manejar protocolos durante la realización de las actividades y no cuando sucedan accidentes para las empresas y operadoras de turismo junto con sus proveedores. Según la Adventure Travel Trade Association existen dos principales categorías: aventura fuerte y aventura suave (Tabla 1).

\section{Tabla 1. Tipología de turismo de aventura}

\begin{tabular}{lll}
\hline & & Actividades \\
\hline Tipo fuerte & Tipo suave & Otros \\
\hline Canotaje & Expedición arqueológica & Crucero \\
Exploración de cuevas & Asistencia a ferias locales & Actividades culturales \\
Ascenso a montañas & Mochilero & Conocer Gente Local \\
Trekking & Observador de aves & Aprender nuevo idioma \\
& Camping & Tour de caminata \\
& Ecoturismo & Visita de amigos/familia \\
& Programas educacionales & Visita de sitios históricos \\
& Actividades ambientales & \\
& Pesca & \\
& Excursionismo & \\
& Equitación & \\
& Cacería & \\
& Kayaking & \\
& Orientación & \\
& Rafting & \\
& Expediciones de búsqueda & \\
\hline
\end{tabular}

Fuente: Elaboración propia basada en Revista Global Report on Adventure Tourism por UNWTO (2014)

En Ecuador dentro del Reglamento de Turismo de Aventura del Ministerio de Turismo (MINTUR, 2014), en su Artículo 6, menciona que las modalidades de turis- mo de aventura se clasifican de acuerdo al elemento natural donde se desarrollan, esto es tierra, aire, agua (Figura 1).

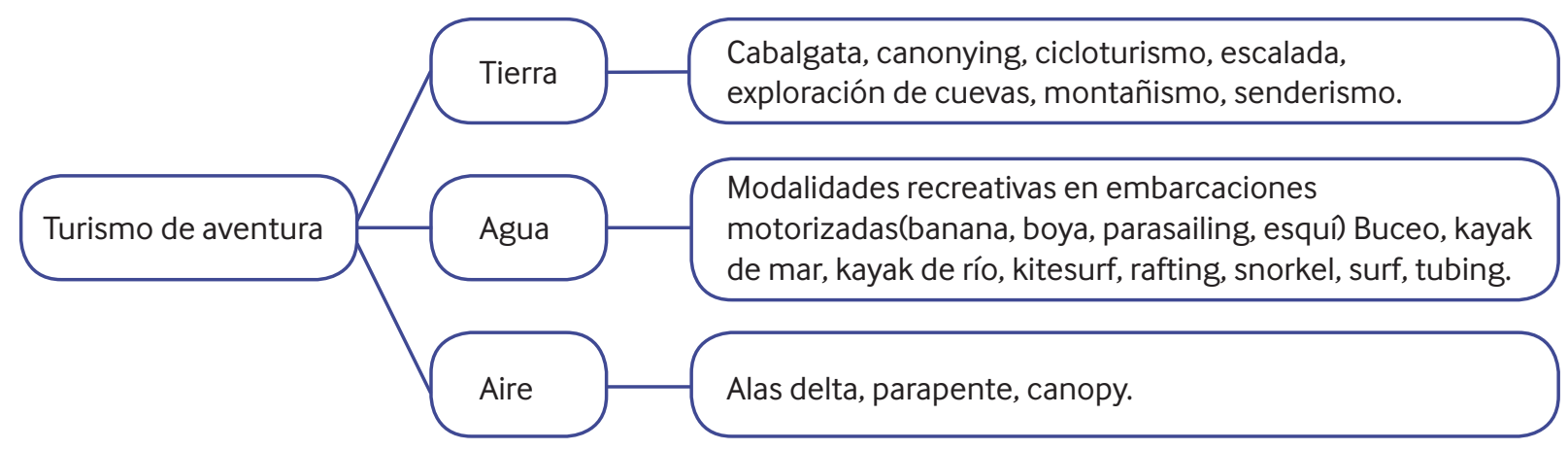

Fuente: Elaboración propia basado en clasificación del Reglamento de Actividades Turísticas (MINTUR, 2014). 
Es importante establecer que un paquete de turismo se define como "el conjunto de ofertas en el que se puede componer de uno o más servicios incluyendo el "todo incluido" elaborado por agencias de viaje". El "todo incluido" es una modalidad de turismo que destaca en los destinos de sol y playa (Corcoran et al., 1996), y se define como itinerarios elaborados por una agencia de viajes, u otro proveedor, donde se incluye al menos tres elementos, como son el transporte, el alojamiento y los traslados, aunque algunos paquetes pueden incluir servicios adicionales como excursiones. En este sentido para Anderson et al., 2009), existen cinco paquetes diferentes, que son paquete básico, alojamiento y desayuno; media pensión, pensión completa y todo incluido, y cada uno de ellos incluiría unos servicios diferentes. Por su parte, Wong \& Kwong (2004), identifica dos tipos de paquetes, el básico y el "todo incluido".

\section{METODOLOGÍA}

La metodología utilizada en la presente investigación se basó en la realización de una encuesta basada en estudios como los de Lázaro (2014), Flecther (2010), y Cole (2016), que sirvieron para recolectar la información acerca de las preferencias y aspectos relacionados con el viaje para crear un producto de turismo de aventura. Para tener un cuestionario que sirva como una herramienta válida, se realizó una prueba piloto a 10 personas para mejorarlo desde el punto de vista técnico y comprensivo. Para obtener los resultados más aproximados se envió el cuestionario a personas que les gusta el turismo de aventura y visitar la provincia de Santa Elena. La presente investigación en lo referente a la toma de la muestra se desarrolló entre los meses de junio a agosto de 2017 en la ciudad de Guayaquil por tener visitantes que viajan a la provincia de Santa Elena y ser un mercado meta muy importante para las operadoras de turismo. Para obtener resultados aproximados se utilizaron preguntas cerradas y de opción múltiple. Se recibieron 223 respuestas, lo que sería la muestra del presente estudio, debido a que no existe un estudio oficial del número de personas que visitan Santa Elena para la práctica del turismo de aventura, se utilizó la ecuación de población infinita donde con un margen de error de $+/-6,5 \%$, un nivel de confianza del $95 \%$ y una varianza del $50 \%$, se pudieron obtener resultados aproximados que sirvieron para conocer las preferencias del turismo de aventura en esta provincia (Tabla 2).

\section{Tabla 2. Ficha de la investigación.}

\section{Población}

Área geográfica

Periodo de realización

Procedimiento

Nivel de confianza

Margen de error

Cuestionarios válidos
Visitantes y turistas de Santa Elena.

Guayaquil.

Junio a agosto del 2017.

Muestreo aleatorio simple.

$95 \%$

$+/-6,5 \%$

223

Fuente: Datos de la investigación.

Los datos, fueron organizados, tabulados y analizados usando el programa SPSS v.22.

Ecuador se encuentra en la parte Noroccidental de América del Sur, el cual cuenta con cuatro regiones naturales: Costa, Sierra, Galápagos y Región Amazónica. Desde la perspectiva económica, en las exportaciones no petroleras, el turismo ocupa el tercer lugar luego del banano y camarón (MINTUR, 2017). Durante el año 2015 el ingreso de divisas por concepto de viajes y transporte de pasajeros alcanzó la suma de 1.557,4 millones de dólares, mientras que en el año 2014 recibió 1.487,2 millones de dólares, observando un incremento del 4,7\% con respecto al año anterior. En lo relacionado a la llegada de turistas, el año 2016 Ecuador recibió 1.412 .718 de turistas internacionales mientras que en el año 2015 recibió 1.543.091 de turistas internacionales.

La provincia de Santa Elena se encuentra situada en el occidente del país, forma parte de la región costa del Ecuador, posee una extensión de $3.763 \mathrm{~km}^{2}$, está constituido por tres cantones que son Santa Elena, La Libertad y Sa- 
linas, con sus respectivas parroquias rurales y urbanas. Conocida por ser cuna de los hermosos balnearios de la costa pacífico, posee una gran variedad de atractivos naturales, culturales e históricos. Los turistas pueden llegar a la ciudad de Guayaquil por el terminal terrestre Jaime Roldós Aguilera o el aeropuerto José Joaquín de Olmedo, para de allí trasladarse vía terrestre a esta provincia que se encuentra a dos horas de distancia, para disfrutar de sus importantes atractivos turísticos. Es importante destacar que a Santa Elena llegan turistas de diferentes provincias del Ecuador y turistas internacionales a disfrutar de su naturaleza y cultura. Posee extensas playas, entre las que destacan Salinas, La Libertad, Chanduy, Ballenita, San Pablo, Palmar, San Pedro, Ayangue, Valdivia, Libertador Bolívar, Manglaralto, Montañita, Olón, Las Núñez y La Entrada. La provincia es atravesada por la ruta del Spondylus que incluye los tres cantones antes mencionados. La provincia de Santa Elena cuenta con una variedad de sitios donde es posible la práctica del turismo de aventura en sus distintas modalidades (Tabla 3).

\section{Tabla 3. Sitios para la práctica del turismo de aventura en Santa Elena.}

\begin{tabular}{ll}
\hline Lugar & \multicolumn{1}{c}{ Actividad } \\
\hline Salinas & Esquíacuático \\
& Parasailing \\
San Pablo & Cicloturismo \\
& Kayak \\
& Kitesurfing \\
Palmar & Senderismo \\
Ayangue & Kayak \\
& Buceo \\
San Pedro & Snorkel \\
Dos Mangas & Parapente \\
& Senderismo \\
Montañita & Cicloturismo \\
& Canopy \\
& Surf \\
Olón & Parapente \\
\hline
\end{tabular}

Fuente: Datos de la investigación.

\section{RESULTADOS Y DISCUSIÓN}

Acerca de los aspectos sociodemográficos en la Tabla 4, se puede observar que la muestra estuvo conformada en lo referente a la edad por el grupo mayoritario de jóvenes de menos de 30 años (76,1\%), seguido del grupo de 3039 años de edad (17,4\%). En cuanto al género el $66,1 \%$ fueron mujeres y un $33,9 \%$ fueron hombres. El estado civil el grupo mayoritario eran solteros $(78,4 \%$ ) y casados $(11,5 \%)$. En lo referente a la formación, el grupo mayo- ritario tenía formación universitaria (78,8\%), seguido de formación secundaria $(11,1 \%)$ y formación de post-grado (10,1\%). En cuanto a la actividad laboral se puede observar que el grupo mayoritario del $56,9 \%$ corresponde a estudiantes, un $18,3 \%$ a empleado privado, un $9,2 \%$ a empleado público, un 7,3\% a profesionales independientes, un $3,2 \%$ a empresarios y ama de casa, y un $2,3 \%$ a jubilados. 
Tabla 4. Variables socioeconómicas

\begin{tabular}{llc}
\hline Descripción & Variable & Porcentaje (\%) \\
\hline Sexo & Hombre & 33,9 \\
Edad & Mujer & 66,1 \\
& Menos de 30 años & 76,1 \\
& $30-39$ años & 17,4 \\
& $40-49$ años & 3,7 \\
Estado Civil & $50-59$ años & 0,9 \\
& 60 años o más & 1,8 \\
& Soltero & 78,4 \\
& Unión libre & 28,0 \\
Nivel de formación & Viudo & 4,6 \\
& Casado & 11,5 \\
& Divorciado & 4,1 \\
& Enseñanza primaria & 0,0 \\
& Enseñanza secundaria & 11,1 \\
Actividad profesional & Enseñanza universitaria & 78,8 \\
& Postgrado/ Maestría/ Ph.D. & 10,1 \\
& Estudiante & 56,9 \\
& Empresario/a privado & 3,2 \\
& Empleado público & 9,2 \\
& Empleado privado & 18,3
\end{tabular}

Fuente: Datos de la investigación.

Sobre las preferencias, el estudio evidencia que a u 76.4\% de personas les gustaría realizar turismo de aventura en sus diversas modalidades, seguido de un $19,4 \%$ que res- pondieron que tal vez realizarían este tipo de turismo, lo que demuestra la importancia de este tipo de turismo en Ecuador (Tabla 5).

Tabla 5. Personas que les gustaría practicar turismo de aventura

\begin{tabular}{lcc}
\hline Opción & Cantidad & Porcentaje (\%) \\
\hline $\mathrm{Si}$ & 165 & 76,4 \\
$\mathrm{No}$ & 9 & 4,2 \\
Tal vez & 42 & 19,4 \\
Total & 223 & $100 \%$ \\
\hline
\end{tabular}

Fuente: Datos de la investigación.

En la Tabla 6 se muestran los resultados sobre las preferencias del tipo de modalidad de turismo de aventura que le gustaría realizar a los encuestados. Se encontró como resultados que entre los principales grupos un $60,6 \%$ de las personas le gustaría realizar parapente; un $48,4 \%$ con- testó que le gustaría realizar buceo; seguido de un 43,2\% correspondiente a snorkel; un 36,2\% le gustaría practicar cicloturismo; un 35,2\% eligió kayak; seguido de un 34,7\% que prefirieron realizar senderismo y un $29,1 \%$ eligió practicar canopy. 
Tabla 6. Preferencias de modalidades de aventura que le gustaría practicar al turista

\begin{tabular}{lccc}
\hline Opción & Cantidad & Porcentaje (\%) & Ranking \\
\hline Parapente & 129 & 60,6 & 1 \\
Buceo & 103 & 48,4 & 2 \\
Snorkel & 92 & 43,2 & 3 \\
Cicloturismo & 77 & 36,2 & 4 \\
Kayak & 75 & 35,2 & 5 \\
Senderismo & 74 & 34,7 & 6 \\
Canopy & 62 & 29,1 & 7 \\
Esquí acuático & 58 & 27,2 & 8 \\
Surf & 50 & 23,5 & 9 \\
Parasailing & 44 & 20,7 & 10 \\
Kitesurfing & 25 & 11,7 & 11 \\
Total & 223 & $100 \%$ & \\
\hline
\end{tabular}

Fuente: Datos de la investigación.

En la Tabla 7, el estudio evidencia la cantidad de dinero que los visitantes están dispuestos a pagar por la compra de un paquete de turismo de aventura. Un $65,1 \%$ optó por la cantidad de 100 a 150 dólares como grupo mayoritario, seguido de un 23,7\% que sugirió pagar entre 151 a 250 dólares.

Tabla 7. Valores dispuestos a pagar por paquete de turismo de aventura

\begin{tabular}{lccc}
\hline Opción & Cantidad & Porcentaje (\%) & Ranking \\
\hline \$100 - \$150 & 140 & 65,1 & 1 \\
$\$ 151-\$ 250$ & 51 & 23,7 & 2 \\
\$251 - \$300 & 7 & 3,3 & 3 \\
\$300 o más & 2 & 0,9 & 4 \\
Total & 223 & $100 \%$ & \\
\hline
\end{tabular}

Fuente: Datos de la investigación.

En la Tabla 8, los resultados muestran el tiempo que el encuestado desearía quedarse realizando turismo de aven- tura. El 42,3\% escogió 3 días y 2 noches siendo el más alto, seguido de un $40 \%$ que corresponde a 2 días y 1 noche.

Tabla 8. Tiempo de estadía

\begin{tabular}{lcc}
\hline Opción & Cantidad & Porcentaje (\%) \\
\hline 2 días - 1 noche & 86 & 40 \\
3 días - 2 noches & 91 & 42,3 \\
4 días - 3 noches & 24 & 11,2 \\
5 días - 4 noches & 8 & 3,7 \\
6 días o más & 6 & 2,8 \\
Total & 223 & $100 \%$ \\
\hline
\end{tabular}

Fuente: Datos de la investigación. 
La modalidad de turismo de aventura que prefirieron realizar los encuestados en la provincia de Santa Elena, se muestran en la Tabla 9. Con un mayor porcentaje se muestra el buceo de Ayangue (46,9\%), seguido de la práctica de snorkel en Ayangue (39\%), parapente en Montañita $(36,2 \%)$, encontrándose como hallazgo que las activida- des con mayor preferencia para el turismo de aventura en la provincia de Santa Elena son buceo, snorkel y parapente. Le siguen esquí acuático en Salinas (32,9\%), canopy en Montañita (30\%) y parapente en San Pedro (30\%). La pregunta era de respuesta múltiple, por lo tanto la sumatoria del resultado es superior a $100 \%$.

Tabla 9. Preferencias sobre las modalidades de aventura en Santa Elena

\begin{tabular}{lccc}
\hline Opción & Cantidad & Porcentaje (\%) & Ranking \\
\hline Buceo en Ayangue & 100 & 46,9 & 1 \\
Snorkel en Ayangue & 83 & 39 & 2 \\
Parapente en Montañita & 77 & 36,2 & 3 \\
Esquí acuático en Salinas & 70 & 32,9 & 4 \\
Canopy en Montañita & 64 & 30 & 5 \\
Parapente en San Pedro & 64 & 30 & 6 \\
Kayak en San Pablo & 54 & 25,4 & 7 \\
Cicloturismo en San Pablo & 52 & 24,4 & 8 \\
Senderismo en Dos Mangas & 52 & 24,4 & 9 \\
Cicloturismo en Dos Mangas & 51 & 23,9 & 10 \\
Senderismo en San Pablo & 44 & 20,7 & 12 \\
Surf en Montañita & 39 & 18,3 & 13 \\
Kayak en Palmar & 32 & 15 & 14 \\
Parasailing en Salinas & 32 & 15 & 15 \\
Kitesurfing en San Pablo & 29 & 13,6 & 16 \\
Surf en Olón & 29 & 13,6 & 17 \\
Parasailing en Montañita & 28 & 13,1 & 12 \\
\hline
\end{tabular}

Fuente: Datos de la investigación.

\section{CONCLUSIONES}

El presente trabajo empírico muestra que estudiar las preferencias para el turismo de aventura en un destino trae información útil para elaborar paquetes turísticos y mejorar la oferta de productos en un destino que cuenta con recursos naturales para el desarrollo de esta modalidad de turismo. Los resultados evidencian información importante acerca de las preferencias del turista de aventura en la provincia de Santa Elena y contribuyen con un modelo para crear paquetes de aventura. Así, el estudio muestra que un $76,4 \%$ de personas les gustaría realizar turismo de aventura en sus diversas modalidades, seguido de un $19,4 \%$ que respondieron que tal vez realizarían este tipo de turismo en los diferentes destinos que los ofrecen.
Acerca de las preferencias en el tipo de turismo de aventura, un $60.6 \%$ de las personas le gustaría realizar parapente, un $48,4 \%$ respondió que le gustaría realizar buceo, seguido de un $43,2 \%$ correspondiente a buceo.

Acerca de las preferencias sobre la modalidad de turismo de aventura que prefirieron realizar los encuestados en la provincia de Santa Elena, los resultados muestran con un mayor porcentaje el buceo de Ayangue (46,9\%) seguido de la práctica de snorkel en Ayangue (39\%), parapente en Montañita (36,2\%) siendo las actividades con mayor preferencia para el turismo de aventura en la provincia de Santa Elena. Le siguen esquí acuático en Salinas (32,9\%), canopy en Montañita (30\%) y parapente en San Pedro 
(30\%). Encontrándose como hallazgo que el buceo que se realiza en la comunidad de Ayangue es el que más aceptación tuvo de todas las modalidades.

Con estos resultados se ha obtenido información para crear paquetes turísticos dirigidos a hombres y mujeres jóvenes de menos de 30 años, solteros universitarios y con ganas de realizar este tipo de modalidades. Las preferencias determinan que desearía estar entre tres días y dos noches, y de dos días con una noche con las modalidades que obtuvieron más porcentaje tales como buceo, snorkel, parapente, canopy y esquí acuático, basado en el precio entre 100 a 150 dólares por paquete turístico, lo cual es accesible para este segmento. La principal limitación de este estudio es el periodo temporal en el que se realizó el trabajo de campo.

\section{REFERENCIAS}

Anderson, W., Juaneda, C., Sastre, F. (2009). Influences of pro-all-inclusive travel decisions. Tourism Review 64, 2, 4-18.

Butler, R.W. (1993). Tourism-an evolutionary perspective. Tourism and Sustainable Development: Monitoring, Planning, Managing 37.

Buckley, R. (2009). Evaluating the net effects of ecotourism on the environment: A framework, first assessment and future research. Journal of Sustainable Tourism 17, 6, 643-672.

Cloke, P., Perkins, H.C. (1998). "Cracking the canyon with the awesome foursome": representations of adventure tourism in New Zealand. Environment and Planning D: Society and Space 16, 2, 185-218.

Cole, Z. (2016). Identifying adventure tourism product signatures: A case analysis of guided mountain bike tours. E-review of Tourism Research 13.

Corcoran, L.M., Gillmor, D.A., Killen, J.E. (1996). An analysis of summer sun tourists-outbound package holidays from Dublin Airport. Irish Geography 29, 2, 106-115.

Díaz Carrión, I.A. (2012). Turismo de aventura y participación de las mujeres en Jalcomulco (México). PASOS Revista de Turismo y Patrimonio Cultural 10, 5.

Fletcher, R. (2010). The emperor's new adventure: Public secrecy and the paradox of adventure tourism. Journal of Contemporary Ethnography 39, 1, 6-33.

Ministerio de Turismo del Ecuador, MINTUR. (2014). Reglamento de Actividades Turísticas de Aventura. Quito, Ecuador: Servicios Publicados.

Saldaña, L.M. (2014). La calidad técnica en el turismo de aventura. Apunts. Educación Física y Deportes 2, 116, 80-86.

Williams, P., Soutar, G.N. (2009). Value, satisfaction and behavioral intentions in an adventure tourism context. Annals of Tourism Research 36, 3, 413-438.

Wong, C.K.S., Kwong, W.Y.Y. (2004). Outbound tourists' selection criteria for choosing all-inclusive package tours. Tourism Management 25, 5, 581-592. 